OPEN ACCESS

Edited by:

Abhay Satoskar,

Ohio State University, USA

Reviewed by:

Takayuki Yoshimoto,

Tokyo Medical University, Japan

Joseph Barbi,

Roswell Park Cancer Institute, USA

${ }^{*}$ Correspondence:

Carlos Penha-Gonçalves

cpenha@igc.gulbenkian.pt

Specialty section:

This article was submitted to

Microbial Immunology,

a section of the journal

Frontiers in Immunology

Received: 16 September 2016

Accepted: 19 January 2017

Published: 06 February 2017

Citation:

Gonçalves LA, Rodo J, Rodrigues-

Duarte L, de Moraes LV and

Penha-Gonçalves C (2017) HGF

Secreted by Activated Kupffer Cells Induces Apoptosis of PlasmodiumInfected Hepatocytes.

Front. Immunol. 8:90.

doi: 10.3389/fimmu.2017.00090

\section{HGF Secreted by Activated Kupffer Cells Induces Apoptosis of Plasmodium-Infected Hepatocytes}

\author{
Lígia Antunes Gonçalves, Joana Rodo, Lurdes Rodrigues-Duarte, \\ Luciana Vieira de Moraes and Carlos Penha-Gonçalves* \\ Instituto Gulbenkian de Ciência, Oeiras, Portugal
}

Malaria liver stage infection is an obligatory parasite development step and represents a population bottleneck in Plasmodium infections, providing an advantageous target for blocking parasite cycle progression. Parasite development inside hepatocytes implies a gross cellular insult evoking innate host responses to counteract intra-hepatocytic infection. Using primary hepatocyte cultures, we investigated the role of Kupffer cell-derived hepatocyte growth factor (HGF) in malaria liver stage infection. We found that Kupffer cells from Plasmodium-infected livers produced high levels of HGF, which trigger apoptosis of infected hepatocytes through a mitochondrial-independent apoptosis pathway. HGF action in infected hepatocyte primary cultures results in a potent reduction of parasite yield by specifically sensitizing hepatocytes carrying established parasite exo-erythrocytic forms to undergo apoptosis. This apoptosis mechanism is distinct from cell death that is spontaneously induced in infected cultures and is governed by Fas signaling modulation through a mitochondrial-dependent apoptosis pathway. This work indicates that HGF and Fas signaling pathways are part of an orchestrated host apoptosis response that occurs during malaria liver stage infection, decreasing the success of infection of individual hepatocytes. Our results raise the hypothesis that paracrine signals derived from Kupffer cell activation are implicated in directing death of hepatocytes infected with the malaria parasite.

Keywords: Plasmodium, Kupffer cells, hepatocytes, HGF, apoptosis, malaria, liver

\section{INTRODUCTION}

Malaria remains a major global health problem. It is estimated that 3.2 billion people are at risk of developing the disease (1), which represents a serious socioeconomic burden in malaria endemic countries (2). The natural infection is initiated by Plasmodium sporozoites that reach the liver and infect hepatocytes-the preferred site of parasite replication and an obligatory step for cycle progression in the mammalian host (3). Through a high-efficacy process of pre-erythrocytic schizogony, one sporozoite develops into a liver stage form that undergoes exponential growth entrenching thousands of merozoites inside the parasitophorous vacuole in each infected hepatocyte (4). Parasite

Abbreviations: HGF, hepatocyte growth factor; KC, Kupffer cell; EEFs, exo-erythrocytic forms; TREM2, triggering receptor expressed in myeloid cells 2; NKT, natural killer T; ACK, ammonium chloride potassium; TUNEL, terminal deoxynucleotidyl transferase dUTP nick end labeling; Ct, cycle threshold; DAPI, 4',6-diamidino-2-phenylindole; NPCs, liver non-parenchymal cells; lpr, lymphoproliferation; FasL, Fas ligand. 
invasion and the expansion of exo-erythrocytic forms (EEFs) inside hepatocytes represent a prominent cellular insult that plausibly elicits host strategies to counteract EEFs development.

Malaria liver stage infection has shown to induce local innate immune responses. Plasmodium infection has been shown to trigger a type I interferon response that mediates an inflammatory reaction in the liver $(5,6)$. Plasmodium RNA was shown to work as a ligand of the cytosolic pattern recognition receptor Mda5, which activates a type I IFN response via the adaptor molecule Mavs and the transcription factors Irf3 and Irf7 (6). This response decreases liver parasite load possibly because type I IFN signaling in myeloid cells contributes to the recruitment of immune cells to the inflammatory foci around infected hepatocytes (6). In fact, recruitment of natural killer T (NKT) cells in sporozoites-infected livers was shown to be dependent on the expression of interferon-alpha/beta receptor alpha chain (5). Another line of evidence indicates that activation of Kupffer cells is a key component in the outcome of malaria liver stage infection. We have shown that pro-inflammatory activation of Kupffer cells by sporozoites is critically regulated by the innate immunity receptor triggering receptor expressed in myeloid cells 2 (TREM2). The level of TREM2 expression in Kupffer cells directly correlates with natural resistance liver stage infection and with reduced hepatocyte parasite yield (7). Nevertheless, the molecular mechanisms by which Kupffer cells control hepatocyte infection are not completely unveiled.

The outcome of liver stage infection results from balancing host defense responses and parasite mechanisms that counteract such responses. In particular, the success of parasite development and growth inside individual hepatocytes is dependent on the ability to sustain host cell survival until late stages of infection (8). In fact, it has been shown that parasite survival depends on inhibition of hepatocyte apoptosis mechanisms, including p53 downregulation and inhibition of mitochondrial-dependent induced cell death $(9,10)$.

Once sporozoites cross the hepatic sinusoidal barrier, they traverse several hepatocytes before invading and establishing an intracellular infection (11). Shifting to the invasive mode requires the action of host factors released during hepatocyte traversing (12). Hepatocyte growth factor (HGF) signaling has been shown to facilitate Plasmodium berghei sporozoite infection in hepatoma cells (13), possibly by conferring resistance to induced cell death (14). Nevertheless, experimental infection of different hepatoma cell lines showed that the induction of HGF signaling did not improve Plasmodium falciparum invasion (15) and was not crucial for infection by P. falciparum or Plasmodium yoelii (16).

We tested the hypothesis that the local innate immune response induced by $P$. berghei liver stage infection was involved in triggering apoptosis of infected hepatocytes. In particular, we investigated whether HGF signaling pathway is involved in apoptosis of infected hepatocytes and takes part in the response of liver innate immune cells to malaria infection. Our results indicate that activation of Kupffer cells by malaria parasites entails the production of HGF that specifically induce apoptosis of infected hepatocytes. This work supports the view that HGF plays multiple roles during malaria liver stage infection.

\section{MATERIALS AND METHODS}

\section{Mice}

All procedures involving laboratory mice were in accordance with national (Portaria 1005/92) and European regulations (European Directive 86/609/CEE) on animal experimentation and were approved by the Instituto Gulbenkian de Ciência Ethics Committee, and the Direcção-Geral de Veterinária (the Official National Entity for regulation of laboratory animals usage). The mice were bred and maintained in conventional housing facilities at the Instituto Gulbenkian de Ciência. All experiments were conducted using male mice with 8-15 weeks of age from strains C57BL/6, BALB/c, and B6. lpr (B6.MRL-Fas $\left.{ }^{l p r} / \mathrm{J}\right)$.

\section{Hepatocyte Primary Cultures}

Mouse primary hepatocytes were prepared as previously described (17). In short, mouse liver lobes were perfused with liver perfusion medium and liver digest medium (Gibco; Invitrogen) at $37^{\circ} \mathrm{C}$. Dissociated cells from the tissue were separated using a 1.12, 1.08, and $1.06 \mathrm{~g} / \mathrm{ml}$ Percoll (GE Healthcare) gradient. Hepatocytes were collected from the gradient solution and cultured in William's E complete medium (Gibco; Invitrogen) in plates or glass coverslips coated with $0.2 \%$ gelatine. The viability of hepatocytes in each experiment preparation was assessed by vital staining with Trypan blue and by cell morphology in FACS analysis. All the experiments were performed with viability of hepatocytes preparations above $80 \%$ purity. The remaining dead cells were removed $12 \mathrm{~h}$ post-plating ( $12 \mathrm{~h}$ prior infection), as in freshly isolated preparations dead hepatocytes do not attach. HGF conditioning was performed by adding $100 \mathrm{ng} / \mathrm{ml}$ of recombinant mouse HGF (eBioscience) $1 \mathrm{~h}$ prior infection, with a subsequent wash before infection.

\section{Parasites and Infection}

Green fluorescent protein $(g f p)$-expressing $P$. berghei ANKA (Pb) (18), P. yoelii 17XNL (Py), and Pb-spect(-)1 (19) sporozoites were obtained after dissection of infected salivary glands from Anopheles stephensi mosquitoes bred in the insectarium of the Instituto de Medicina Molecular, Lisbon. Briefly, mosquitoes were immobilized, and the thorax and abdomen were separated with fine forceps. The salivary glands were dissected from the thoraces into RPMI medium, collected to a $1.5 \mathrm{ml}$ Eppendorf, and smashed to release the sporozoites. The sporozoites were collected from the supernatant upon a $2 \mathrm{~min} \times 5 \mathrm{~min} 800 \mathrm{rpm}$ centrifugation (20). The same procedure was applied to non-infected mosquitoes to obtain salivary glands suspensions. Mice were intravenously inoculated with $10^{4}$ sporozoites each. Hepatocytes were infected with 1.5 to $3.5 \times 10^{4}$ sporozoites, and non-infected controls were mock infected with salivary glands suspensions of non-infected mosquitoes.

\section{Isolation of Liver Non-Parenchymal Cells (NPCs)}

Non-parenchymal cells were isolated from mouse livers at $40 \mathrm{~h}$ post-infection. Cells were obtained by adapting a method previously described (21). Briefly, liver lobes were removed 
and perfused with liver perfusion medium (Gibco; Invitrogen) with $750 \mathrm{mg} / \mathrm{l}$ of Collagenase $\mathrm{H}$ (Roche) at $37^{\circ} \mathrm{C}$. The resulting suspension was filtered through a $100 \mu \mathrm{m}$ cell strainer (BD Falcon). The dissociated cells were suspended in liver perfusion medium and centrifuged for $10 \mathrm{~min}$ at $1,500 \mathrm{rpm}$, resuspended in RPMI complete medium (Gibco; Invitrogen), mixed in Percoll (GE Healthcare) solution to give a final concentration of $30 \%$ Percoll, and then centrifuged at 2,000 rpm for $10 \mathrm{~min}$. The cell pellet was resuspended in RPMI and carefully laid on $30 \%$ Percoll solution and centrifuged at 2,000 rpm for $10 \mathrm{~min}$. The cell pellet collected was washed and resuspended in ACK $\left(\mathrm{NH}_{4} \mathrm{Cl} 0.15 \mathrm{M}, \mathrm{KHCO}_{3} 10 \mathrm{mM}, \mathrm{Na}_{2} \mathrm{EDTA} \cdot 2 \mathrm{H}_{2} \mathrm{O} 0.1 \mathrm{mM}\right.$ and $\mathrm{pH} \mathrm{7.2)} \mathrm{for} 3 \mathrm{~min}$ to lyse remaining erythrocytes. Cells were washed and centrifuged at $800 \mathrm{rpm}$ for $20 \mathrm{~s}$ to discard the remaining hepatocytes; the supernatant was recovered, and NPCs were collected at $1,500 \mathrm{rpm}$ for $5 \mathrm{~min}$.

\section{Total RNA and cDNA Synthesis}

Livers and NPCs were collected at $40 \mathrm{~h}$ post-infection, immediately homogenized in denaturing solution and total RNA was obtained using High Pure RNA Tissue Kit and High Pure RNA Kit (Roche). One microgram of total RNA was converted to cDNA (Transcriptor First Strand cDNA Synthesis Kit; Roche). Hepatocytes in culture were collected at $24 \mathrm{~h}$ post-infection, the lysis and reverse transcriptase reaction were performed using TaqMan Gene Expression Cell-to-CT kit (Ambion).

\section{Plasmodium RNA Quantification and Hepatocyte Gene Expression}

cDNA specific to $P$. berghei $18 \mathrm{~S}$ rRNA was amplified by using the Taqman specific primers: IGC-Pb1 (forward) 5'-CCGATAACGAACGAGATCTTAACCT-3', IGC-Pb2 (reverse) 5' -CGTCAAAACCAATCTCCCAATAAAGG-3' and IGC$\mathrm{Pb} 3$ (specific fluorogenic probe) 5'-FAM-ACTCGCCGCTAAT TAG-MGB-3' (7). cDNA specific to P. yoelii $18 \mathrm{~S}$ rRNA was amplified by using the SYBR green system and the specific primers NYU-Py3 5'-GGGGATTGGTTTTGACGTTTTTGCG-3' and NYU-Py55'-AAGCATTAAATAAAGCGAATACATCCTTAT-3' (22). Hgf, Bid, Casp3, Hifo, Pik3r1, Cflar, Fadd, and Casp8 expression was quantified using TaqMan Gene Expression Assays from ABI (Mm01135185_m1, Mm00432073_m1, Mm01195084_m1, Mm00468872_m1, Mm01282780, Mm01255576_m1, Mm00438861_m1, and Mm00802247_m1, respectively). Endogenous control Gapdh (Mouse GAPD Endogenous Control; ABI) was used in multiplex PCR with target genes. PCR reactions were performed with ABI Prism 7900HT, and relative quantification was calculated by the $\Delta \Delta \mathrm{Ct}$ method, using the mean of the control group as calibrator.

\section{Hepatocyte Immunocytochemistry: TUNEL, EEFs, and FasL}

Hepatocytes cultured on coverslips were fixed 24 or 40 h postinfection with $4 \%$ paraformaldehyde (PFA) and incubated with $50 \mathrm{nM} \mathrm{NH}_{4} \mathrm{Cl}$ to extinguish PFA activity. Cells were permeabilized and blocked with blocking solution for $1 \mathrm{~h}$ [3\% of Bovine Serum Albumin (Calbiochem), $100 \mathrm{mM}$ of glycine, $0.1 \%$ of saponin, and $10 \%$ of Fetal Calf Serum (Gibco; Invitrogen) in PBS $1 \times]$ and stained for apoptosis by Terminal Deoxynucleotidyl Transferase dUTP Nick End Labeling (TUNEL) using "In Situ Cell Death Detection-TMR" (Roche); to stain EEFs coverslips were then incubated $45 \mathrm{~min}$ at room temperature with blocking solution containing anti-GFP IgG Alexa-488 conjugated antibody (Invitrogen); followed by DAPI staining (Invitrogen) and mounted with MOWIOL (Calbiochem); to stain FasL, cells were incubated $45 \mathrm{~min}$ at room temperature with blocking solution containing anti-FasL (CD178.1 Kay10; BD Pharmingen) followed by DAPI staining (Invitrogen) and mounted with MOWIOL (Calbiochem). Cell culture images were acquired using an automated Nikon Eclipse TE2000-S inverted fluorescence microscope, with a $20 \times$ objective, covering the total coverslip area. Image $(\mathrm{NIH})$ software was used to differentially identify TUNEL-positive or -negative (live) cells, EEFs and FasL in each coverslip, and to estimate single EEFs area. Results are expressed as the number of TUNEL-positive cells and live cells with EEFs per 100 microscopic fields, or FasL ${ }^{+}$cells per $10^{3}$ cells.

\section{Kupffer Cells FACS Analysis and Purification}

Liver macrophages were stained, at $4^{\circ} \mathrm{C}$, by incubating liver NPCs with F4/80 APC (A3-1; Serotec), CD11c PE (HL3; BD Pharmingen), and CD49b Biotin (DX5 and streptavidin-PerCP; BD Pharmigen) antibodies. Subsequently, a two-step HGF intracellular staining (H170; Santa Cruz and goat anti-rabbit IgG-FITC) was performed by using the BD Cytofix/Cytoperm kit. FACScalibur cytofluorometer (Becton Dickinson) and FlowJo software were used for FACS analysis. Purified Kupffer cells were obtained through high-speed cell sorting (FACSAria; Becton Dickinson), after staining with F4/80 APC (Serotec).

\section{Kupffer Cell-Hepatocyte Transwell Cultures}

We used a $0.4-\mu \mathrm{m}$ transwell culture system (Millipore) to allow purified Kupffer cells (upper chamber) to exchange soluble factors during $12 \mathrm{~h}$ with primary hepatocyte cultures (lower chamber), before infection. Hepatocytes were then infected with $P$. berghei ANKA sporozoites and fixed for parasite and apoptosis staining or collected for RNA analysis at $24 \mathrm{~h}$ post-infection. For HGF/ MET signaling blocking, we added anti-Met antibodies (N-17; Santa Cruz Biotechnology) at $400 \mathrm{ng} / \mathrm{ml}$ when purified Kupffer cells were put in culture with primary hepatocytes followed by an additional $400 \mathrm{ng} / \mathrm{ml}$ added $1 \mathrm{~h}$ prior infection.

\section{HGF ELISA}

Hepatocyte growth factor secretion in the supernatants from transwell cultures was evaluated by sandwich ELISA. Briefly, maxisorp plates were coated with a capture anti-HGF antibody (H-170; Santa Cruz) in 0.5 M Carbonate Bicarbonate Solution. Detection used anti-HGF-biotinylated antibody (BAF2207; R\&D). Samples were quantified against a standard curve with mouse recombinant HGF (eBioscience). 


\section{Fas Pathway Modulation}

Fas-blocking experiments were performed by using either a blocking antibody or a pan-caspase inhibitor. We used $10 \mu \mathrm{g} /$ $\mathrm{ml}$ of anti-FasL antibody (CD178.1 Kay10; BD Biosciences) and $20 \mu \mathrm{M}$ of Z-VAD-FMK (pan-caspase inhibitor - 550377; BD Biosciences), added $1 \mathrm{~h}$ prior infection and using the appropriate controls. Fas signaling was induced with $1 \mu \mathrm{g} / \mathrm{ml}$ of anti-Fas antibody (CD95 Jo2; BD Biosciences) $18 \mathrm{~h}$ post-infection with P. berghei. Cflar knockdown was achieved through a mixture of three distinct siRNAs (s63907, s63908, and s63909; Ambion), at a concentration of $20 \mu \mathrm{M}$ that was delivered to primary hepatocytes with Oligofectamine (Invitrogen). Control samples included untransfected cells and cells transfected with a negative control siRNA (Ambion) or with a positive control targeting mouse Gapdh (Ambion).

\section{Statistical Analysis}

All experiments were performed with a minimum of triplicate samples, and data are representative of at least three independent experiments. Results are presented as average values and error bars represent SD. Statistical analysis used a Mann-Whitney test, and $p<0.05$ was considered statistically significant.

\section{RESULTS}

\section{HGF Impacts on the Yield of Plasmodium Liver Stage Infection}

Liver NPCs, specifically Kupffer cells, have a role in the natural control of Plasmodium liver stage expansion (7). We found that $H g f$ mRNA expression in isolated non-parenchymal liver cells was highly increased upon in vivo infection with $P$. berghei sporozoites (Figure 1A) and correlated with relative resistance to parasite liver stage expansion in BALB/c as compared to C57BL/6 mice (Figure 1B). It has been reported that HGF and its mRNA localize exclusively to NPCs in the liver (23-25), and we have confirmed that expression of $H g f$ mRNA in primary hepatocytes was not detectable, irrespective to infection with $P$. berghei (data not shown). Given that HGF has been shown to facilitate liver stage infection $(13,14,16)$, we investigated the cellular source of HGF in infected livers. FACS analysis of NPCs isolated from sporozoite-infected mice revealed that $\mathrm{F} 4 / 80^{+}$cells, representing Kupffer cells, expressed intracellular HGF as opposed to other NPCs, e.g., CD11 $c^{+}$(dendritic cells) or $\mathrm{CD}_{4} 9 \mathrm{~b}^{+}$cells that include NKT and NK cells (Figure 1C). Furthermore, we found that depletion of liver macrophages abrogated the augmented expression of HGF in the liver induced by infection (Figure S1 in Supplementary Material) suggesting that liver macrophages are the main in vivo source of $\mathrm{HGF}$ in malaria liver stage infection. To untangle the effect of HGF in Plasmodium liver stage infection, we pre-treated hepatocyte primary cultures of C57BL/6 and $\mathrm{BALB} / \mathrm{c}$ mice with HGF $1 \mathrm{~h}$ prior infection and observed that HGF conditioning similarly impairs parasite yield in both mouse strains (Figure 1D). Moreover, HGF pre-treatment of C57BL/6 hepatocytes infected with $P$. yoelii (Figure 1E) or $P$. berghei-spect(-)1 (Figure 1F) revealed that reduction of parasite yield induced by HGF transcends Plasmodium species and is independent of hepatocyte traversing (19). In addition, we found that reduction of parasite burden by HGF was attributable to a significant decrease in the number of hepatocytes carrying EEFs (Figure 1G), detectable from $24 \mathrm{~h}$ post-infection onward. In contrast, HGF conditioning was not associated with altered EEFs size, neither at earlier stages $(24 \mathrm{~h})$ (Figure $\mathbf{1 H})$ nor at the end of the liver infection ( $40 \mathrm{~h}$ ) (Figure 1I). These results show that HGF conditioning reduces the number of hepatocytes that carry EEFs, rather than affecting its development suggesting that Kupffer cell-derived HGF impacts on the survival of infected hepatocytes.

\section{HGF Promotes Apoptosis of EEF-Carrying Hepatocytes}

We investigated whether the reduced number of developed EEFs upon HGF pre-treatment was due to apoptosis of infected hepatocytes. Analysis of mRNA expression of key informative genes showed that HGF induces downregulation of PI3K a component of the HGF/c-Met pathway that is involved in apoptosis protection $(26,27)$ (Figure 2A). Moreover, HGF downregulated Hif1-alpha that has been associated with hepatocyte apoptosis and efficiency of $P$. berghei infection $(28,29)$ (Figure 2B). Furthermore, upregulation of Caspase 3 (Figure 2C), with a concomitant decrease of Bid expression (Figure 2D), suggests that HGF acts through a mitochondrial-independent apoptosis pathway in response to hepatocyte Plasmodium infection (30).

Interestingly, we observed that HGF pre-treatment specifically increased apoptosis susceptibility in parasitized hepatocytes but did not affect bulk apoptosis in the infected cultures (Figures 2E,F), as detected by TUNEL assays. Remarkably, in HGF pre-treated cultures, the size distribution of EEFs inside apoptotic hepatocytes significantly deviated to higher values ( $p$-value $<0.0001$ ) (Figure 2G). Thus, in untreated infected cultures, hepatocytes carrying small EEFs were the major target of spontaneous apoptosis, but under HGF conditioning significant apoptosis was observed in infected hepatocytes with larger EEFs (Figures 2G,H). Collectively, these data indicate that parasitized primary hepatocytes respond to HGF treatment through a mitochondrial-independent apoptosis mechanism, which operates in hepatocytes carrying early liver schizont stages that are refractory to spontaneous apoptosis.

\section{Kupffer Cell-Derived HGF and Efficiency of Primary Hepatocyte Infection}

We ask whether HGF secreted by Kupffer cells could impair hepatocyte infection in vitro. We performed transwell co-culture experiments using FACS sorted $\mathrm{F} 4 / 80^{+}$cells from infected livers and naïve primary hepatocytes. Remarkably, we observed that parasite yield and the number of infected hepatocytes were reduced in the presence of Kupffer cells, to an extent comparable to the cultures pre-treated with HGF (Figures $\mathbf{3 A}, \mathbf{B}$ ), indicating that this effect is attributable to soluble factors derived from Kupffer cells. Moreover, HGF was detected in the supernatants of Kupffer cells/hepatocyte cultures (Figure 3C), which were able to reduce parasite yield when used as media in pure hepatocyte cultures (Figure 3D). Furthermore, adding anti-MET antibodies in the co-cultures to block the action of Kupffer cells-derived 

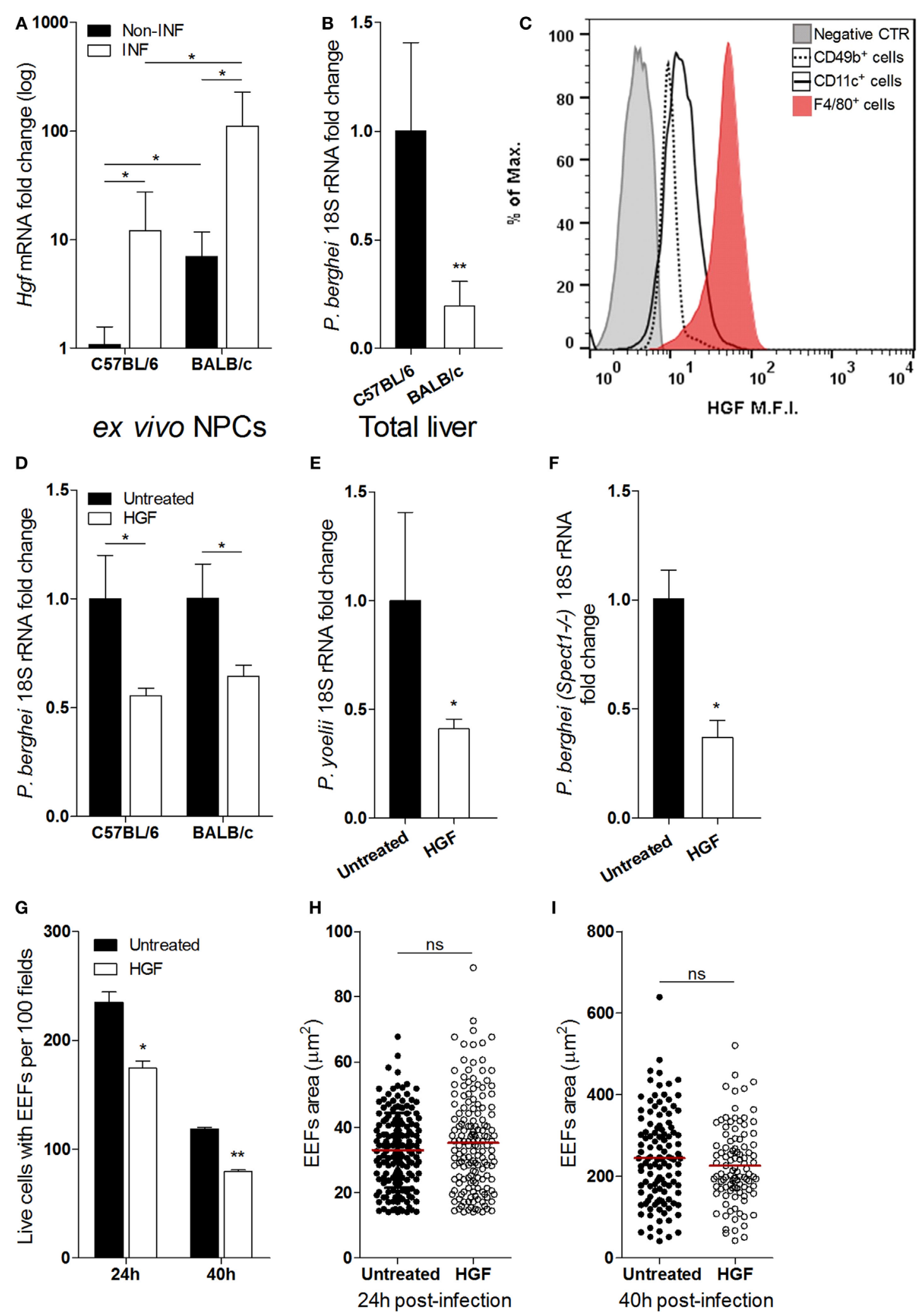

FIGURE 1 | Continued 
FIGURE 1 | Continued

Hepatocyte growth factor (HGF) and yield of malaria liver stage infection in vivo and in vitro. Differential resistance to malaria liver stage infection in C57/ BL/6 and BALB/c mice correlates with increase of HGF expression in non-parenchymal cells (NPCs) (A,B): (A) Hgf mRNA quantification in liver NPCs preparations of infected and non-infected mice and (B) liver parasite burden measured by qRT-PCR of Plasmodium berghei $18 \mathrm{~S}$ rRNA, measured at $40 \mathrm{~h}$ p.i. with P. berghei sporozoites. FACS analysis of liver NPCs isolated from C57BL/6-infected mice revealed high HGF intracellular expression in F4/80+ cells (C). HGF pre-treatment of hepatocyte primary cultures infected with $P$. berghei ANKA reduced malaria liver stage parasite yield in C57BL/6 and BALB/c hepatocytes (D) and in C57BL/6 hepatocytes infected with Plasmodium yoelii (E) or Spect1 deficient $P$. berghei (F), at $24 \mathrm{~h}$ p.i. Effect of HGF pre-treatment in the counts of viable hepatocytes containing exo-erythrocytic forms (EEFs) $\mathbf{( G )}$ and EEFs size (H,I), at 24 and $40 \mathrm{~h}$ p.i. in C57BL/6 hepatocyte primary cultures (non-parametric Mann-Whitney test, ${ }^{*} p<0.05$ and $\left.{ }^{* *} p \leq 0.001\right)$. Data are represented as mean $\pm \mathrm{SD}$ of three livers per mouse strain or triplicate cultures that were performed in at least three independent experiments.

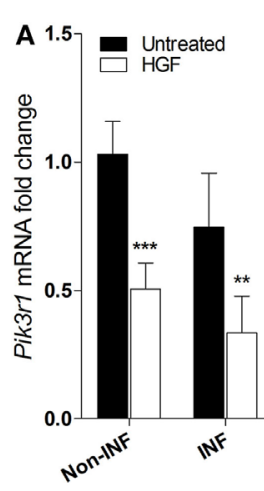

E

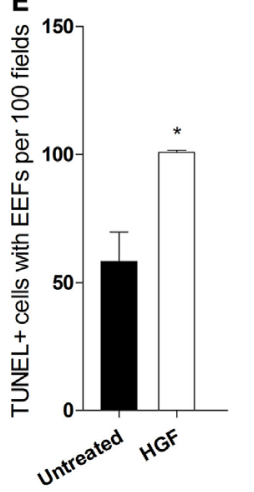

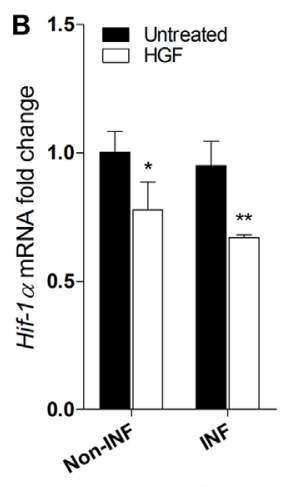

F

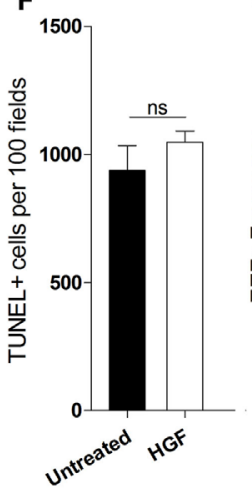

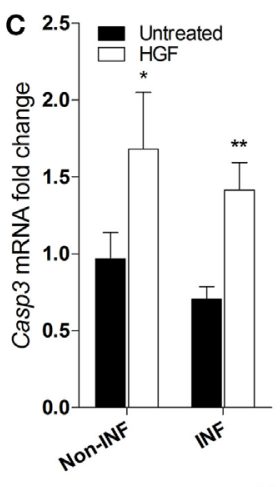

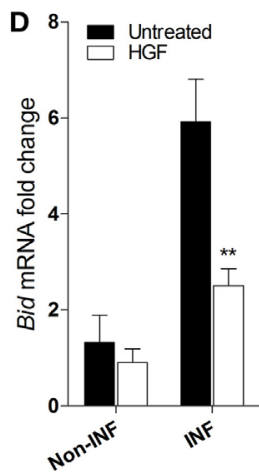

H

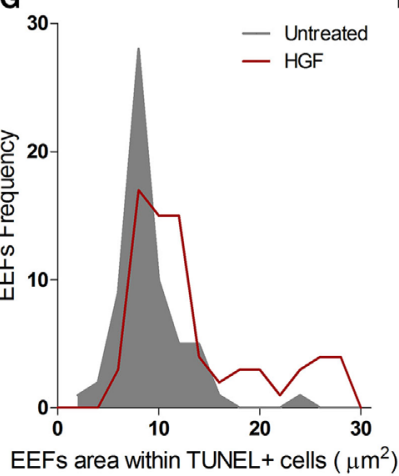

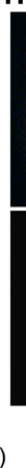

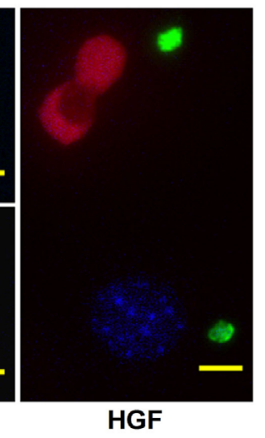

FIGURE 2 | Hepatocyte growth factor (HGF)-conditioning targets exo-erythrocytic form (EEF)-containing hepatocytes for mitochondrial-independent apoptosis. Effect of HGF on the mRNA expression of selected apoptosis-related markers Pik3r1 (A), Hif- $\alpha$ (B), Caspase 3 (C), and Bid (D) in infected (INF) and non-infected (non-INF) hepatocyte cultures at $24 \mathrm{~h}$ p.i. Results represent qRT-PCR estimates relative to non-infected untreated hepatocyte cultures. Effects of HGF pre-treatment on the viability of primary hepatocytes at $24 \mathrm{~h}$ p.i. with Plasmodium berghei sporozoites: (E) apoptotic cells containing EEFs, (F) total apoptotic cells (TUNEL; TUNEL-positive), and (G) size distribution of EEFs harbored in apoptotic cells. (H) Representative immunofluorescence images of primary hepatocyte cultures in the presence (right) or absence (left) of HGF pre-treatment, evidencing larger EEFs in TUNEL + cells after HGF pre-treatment (TUNEL-positive nuclei in red, DAPI-positive in blue and EEFs in green; scale bar $10 \mu \mathrm{m}$ ) (non-parametric Mann-Whitney test, ${ }^{\star} p<0.05$, ${ }^{\star *} p \leq 0.001$, and $\left.{ }^{* \star *} p \leq 0.0001\right)$. Data are represented as mean \pm SD of triplicate cultures that were performed in at least three independent experiments.

HGF led to increased parasite yield in hepatocytes (Figure 3E). Altogether, these results strongly suggest that HGF is secreted by Kupffer cells and acts in an endocrine fashion to reduce successful progression of infection in parasitized hepatocytes.

\section{Involvement of Fas-Mediated Apoptosis in Plasmodium Liver Stage Infection}

Additionally, it has been described that the failure of EEFs to attain full development in vitro is attributable to an hepatocyte autonomous mitochondrial-dependent apoptosis response (10). In line with these observations, we asked whether reduction of the number of EEFs in infected primary hepatocyte cultures occurs as well in the absence of HGF. To ascertain the success of $P$. berghei sporozoites infection in hepatocyte primary cultures, we performed time course counting of infected hepatocytes, which showed that less than $50 \%$ of the initial infected cells attained EEF full development (Figure 4A). To investigate whether the aborted EEFs were related to apoptosis induction in P. bergheiinfected cultures, we analyzed the expression of apoptosis genes 

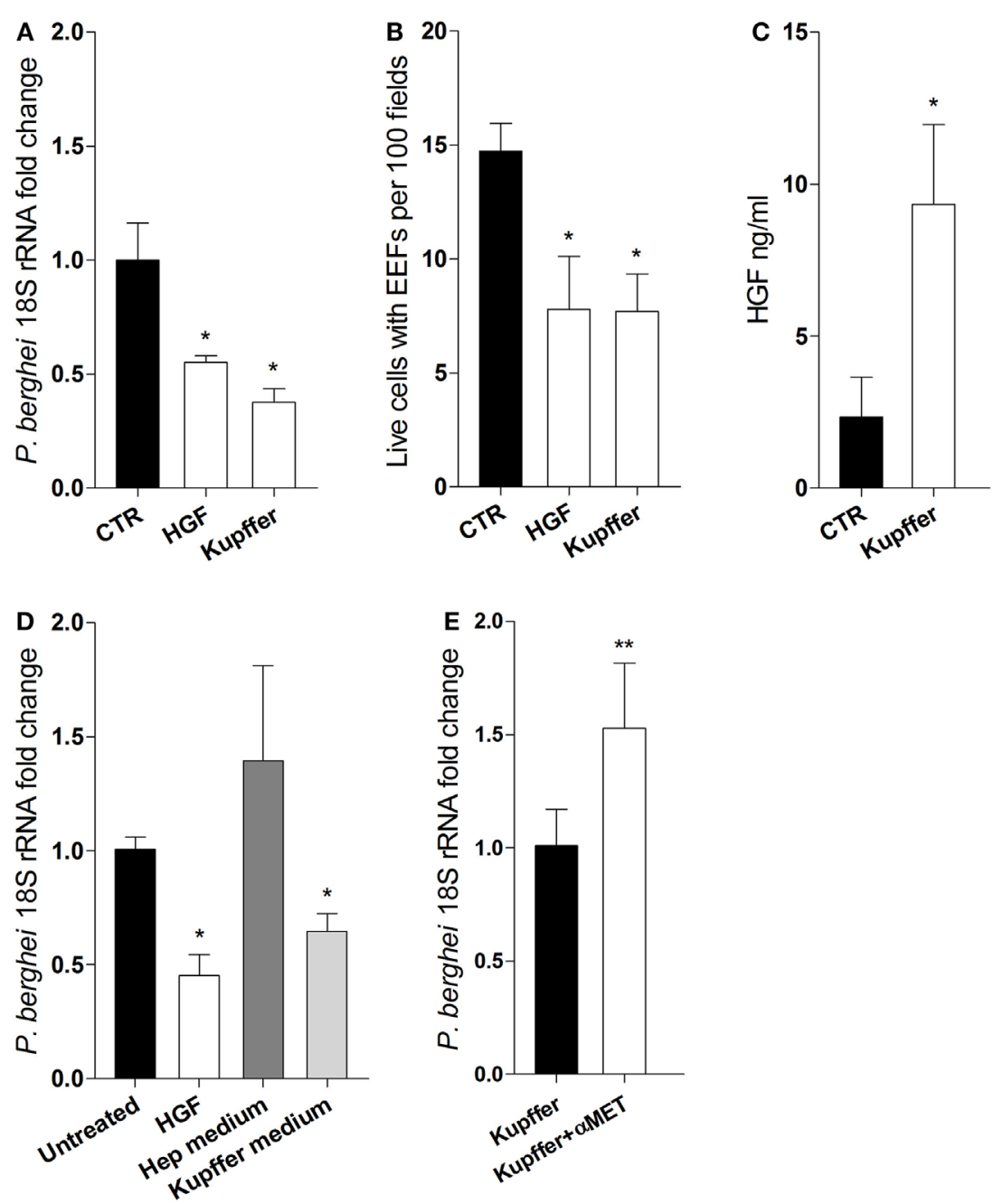

FIGURE 3 | Kupffer cells secrete hepatocyte growth factor (HGF) and control efficacy of primary hepatocyte infection. Transwell co-cultures of liver $\mathrm{F} 4 / 80^{+}$cells (upper chamber) and primary hepatocytes (lower chamber) were compared at $24 \mathrm{~h}$ p.i. (Kupffer), with single hepatocytes cultures pre-treated with HGF (HGF) or left untreated (CTR), for parasite yield (A) and number of viable hepatocytes containing exo-erythrocytic forms (EEFs) (B). Secreted HGF was evaluated in the supernatant of untreated hepatocytes and co-cultures after infection (C). Parasite yield in hepatocyte cultures treated with HGF or incubated with culture supernatant from previous single hepatocytes culture (Hep medium) or from previous hepatocyte-F4/80+ cells co-culture in transwell (Kupffer medium) (D). Parasite yield at $24 \mathrm{~h}$ p.i. in transwell co-cultures of liver F $4 / 80^{+}$cells and primary hepatocytes in the absence (Kupffer) or presence (Kupffer $\left.+\alpha M E T\right)$ of anti-MET blocking antibody (E) (non-parametric Mann-Whitney test, ${ }^{*} p<0.05$ and ${ }^{* *} p \leq 0.001$ ). Data are represented as mean \pm SD of triplicate cultures that were performed in at least three independent experiments, except (E) that represents cumulative data of two independent experiments with triplicate samples.

in hepatocyte primary cultures and found out that Fas pathway signaling-related genes were significantly altered at $24 \mathrm{~h}$ p.i. A strong upregulation of Bid coupled with a slight upregulation of other proapoptotic genes and downregulation of the anti-apoptotic Cflar, represent a signature that Fas pathway signaling was induced in infected primary cultures (Figure 4B), presumably executing a Type II apoptotic response that requires mitochondrial participation (31). To test whether Fas pathway signaling was detrimental to successful hepatocyte infection, we analyzed primary hepatocytes from $l p r$ mice (Fas-null mutant), which demonstrated that at $24 \mathrm{~h}$ p.i. the parasite yield was significantly increased in the absence of Fas pathway signaling. An effect that was also observed by blocking Fas pathway signaling with anti-Fas
Ligand antibodies or with a pan-Caspase inhibitor (Figure 4C). Furthermore, the absence of Fas pathway signaling improved the number of successfully infected hepatocytes (Figure 4D), possibly because apoptosis induced by infection was partially dependent on Fas pathway signaling (Figure 4E). Interestingly, immunofluorescence microscopy of infected cultures revealed prominent FasL expression in non-infected hepatocytes as compared to infected hepatocytes (Figure 4F), indicating that induction of FasL expression by P. berghei infection (Figure 4G) impacts the viability of both infected and non-infected hepatocytes. Conversely, enhancing Fas pathway signaling in hepatocytes either by adding anti-Fas antibodies or by knocking down Cflar, a potent Fas pathway inhibitor (32), increased apoptosis 

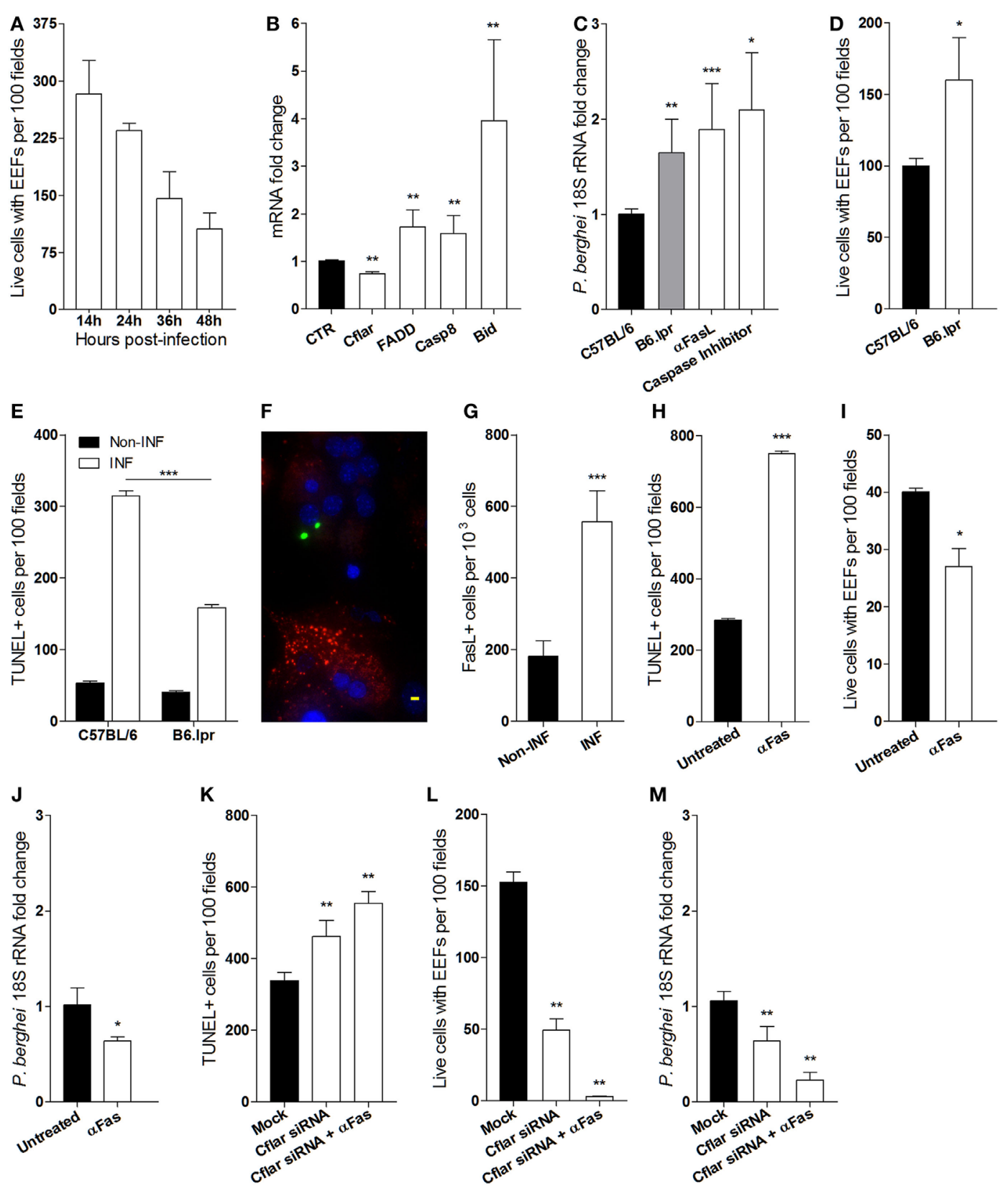

FIGURE 4 | Fas-mediated hepatocyte apoptosis impairs the success of Plasmodium berghei infection (A) exo-erythrocytic form (EEF)-containing viable hepatocytes were counted at indicated times in infected primary cultures. (B) mRNA quantification of Fas pathway genes at $24 \mathrm{~h}$ p.i., relative to non-infected control (CTR) hepatocyte cultures. (C) Parasite yield in infected cultures of Fas-deficient hepatocytes from B6. Ipr mice or after blocking Fas pathway with anti-FasL antibody was measured by qRT-PCR of $P$. berghei $18 \mathrm{~S}$ rRNA at $40 \mathrm{~h}$ p.i. (D) EEF-containing viable hepatocytes of B6. Ipr and C57BL/6 mice were counted and (E) the number of apoptotic cells was determined by TUNEL staining in infected and non-infected cultures at 24 h p.i. (F) Representative immunofluorescence image of non-infected hepatocyte expressing FasL (red) in the vicinity of $P$. berghei-gfp (green) infected hepatocytes [nuclei stained with DAPI (blue)], scale bar-20 $\mu \mathrm{m}$ and (G) counting of FasL-expressing cells in infected and non-infected cultures at $24 \mathrm{~h}$ p.i. (H,K) number of apoptotic cells (TUNELpositive), (I,L) number of viable hepatocytes containing EEFs, and $\mathbf{( J , M ) ~ p a r a s i t e ~ y i e l d ~ w e r e ~ m e a s u r e d ~ i n ~ p r i m a r y ~ h e p a t o c y t e ~ c u l t u r e s ~ i n ~ t h e ~ p r e s e n c e ~ o r ~ a b s e n c e ~ o f ~}$ signaling by anti-Fas antibody and/or Cflar siRNA (non-parametric Mann-Whitney test, ${ }^{*} p<0.05,{ }^{* *} p \leq 0.001$, and ${ }^{* * *} p \leq 0.0001$ ). Data are represented as mean $\pm \mathrm{SD}$ of triplicate cultures that were performed in at least three independent experiments.

susceptibility in infected hepatocyte cultures (Figures $\mathbf{4 H , K}$ ). In these conditions, we observed decreased number of hepatocytes containing EEFs (Figures 4I,L) and reduced parasite yield (Figures 4J,M). The effects of Cflar knockdown were exacerbated by anti-Fas stimulation (Figures $4 \mathbf{K}-\mathbf{M}$ ) and strongly suggest that Cflar inhibition sensitized hepatocytes to undergo apoptosis through the Fas pathway. These experiments provide evidence that apoptosis signals evoked by the parasite favor Fas-dependent 
hepatocyte apoptosis in infected cultures leading to a decrease in the number of infected primary hepatocytes that support full parasite development.

\section{DISCUSSION}

This work revealed an unpredicted hepatocyte-Kupffer cell crosstalk mediated by HGF, which sensitizes Plasmodiuminfected hepatocytes to undergo apoptosis. Furthermore, we provide evidence that Fas-mediated hepatocyte apoptosis is spontaneously induced in infected cultures. Together, the results support the notion that hepatocyte response to Plasmodium in vitro infection entails two distinct apoptosis mechanisms: (1) an HGF-stimulated mitochondrial-independent response that preferably targets hepatocytes harboring early liver schizonts and (2) a Fas-mediated mitochondrial-dependent apoptosis mechanism, acting on both infected and non-infected hepatocytes. Our results suggest that these two responses operate at distinct phases of hepatocyte infection and use different genetic programs for apoptosis induction, representing diverse modes to control malaria liver stage infection.

We have previously shown that expression of innate immunity receptors in Kupffer cells, namely, TREM2, is involved in the genetic resistance to malaria liver stage infection in mouse models (7). We now demonstrate an additional mechanism of parasite yield control mediated by Kupffer cells that specifically targets infected hepatocytes in culture. After in vivo infection, HGF is upregulated in the liver and is mainly expressed by Kupffer cells. This is in line with the notion that HGF and its mRNA localize exclusively to non-parenchymal liver cells (23), namely, Kupffer cells (24). Furthermore, increased HGF mRNA expression in the liver is accompanied by a rise in circulating HGF (33), suggesting an endocrine mechanism of action. Expression of HGF by Kupffer cells has been observed in the context of liver repair and regeneration (34), and our in vitro data indicate that HGF also acts on parasitized hepatocytes to induce apoptosis. These observations are in line with a dual role of HGF in promoting either hepatocyte apoptosis or proliferation depending on the contextual cues $(28,34,35)$. In fact, it was reported that HGF signaling in hepatoma cell lines favors sporozoite transition to the invasive mode and cell survival upon Plasmodium sporozoite infection, in a species-specific fashion $(13,14,16)$. However, our data imply that HGF also has a role in controlling infection by inducing apoptosis of parasitized hepatocytes through a mechanism that transcends Plasmodium species and does not require parasite traversing. Our results embody the hypothesis that HGF produced by infection-activated Kupffer cells, sensitizes primary hepatocytes to engage a mitochondrial-independent apoptosis program (Figures 2A-D) in parasitized cells with established EEFs (Figures $2 \mathrm{E}-\mathrm{H}$ ), potentially overcoming parasite strategies to block spontaneous apoptosis (9).

On the other hand, we observed that infected hepatocyte cultures exhibited spontaneous apoptosis, possibly explaining the reduction of infected cells number in the course of in vitro infection. In line with previous reports (10), we found that inhibition of Fas pathway significantly decreased apoptosis in infected cultures while increasing parasite yield, suggesting that susceptibility of infected hepatocytes to Fas-mediated apoptosis is an efficient way to control initial parasite liver stage infection. Coherently, stimulation of signaling through the Fas pathway decreased the number of infected hepatocytes that supported successful infection. Intriguingly, FasL appears to be overproduced by non-infected hepatocytes that surround individual infected hepatocytes, suggesting that FasL-expressing cells provide a paracrine signal to induce apoptosis of the infected cells (Figure 4). Although susceptibility to Fas-mediated apoptosis plays a role in reducing parasite yield, this effect appeared to affect equally infected and non-infected hepatocytes in the infected cultures. In contrast, HGF signaling promotes a shift from mitochondrialdependent to mitochondrial-independent apoptosis (30) and preferentially targets infected cells in primary cultures. It remains to be investigated what are the intracellular requirements that license apoptosis by each of the two apoptosis mechanisms in order to disentangle their role in the time course of hepatocyte infection.

The decision of whether one infected hepatocyte undergoes apoptosis or support full parasite development might rely on time windows of host apoptosis induction, which is counteracted by effective parasite apoptosis-inhibiting activity (9). In fact, treatment with proapoptotic drugs in vivo was shown to delay or abrogate transition to blood stage infection (36). We have noted that the size of the EEFs found in TUNEL-positive cells in nonsensitized cultures represent early forms of liver stage parasite, suggesting that spontaneous hepatocyte apoptosis is able to deter parasite development at early stages. In contrast, HGF treatment was capable of inducing apoptosis in hepatocytes containing EEFs of larger sizes, indicating that this apoptosis pathway is effective at later stages of parasite development. Together, these observations indicate that the fate of infected hepatocytes is dictated by paracrine interactions occurring between Kupffer cells and hepatocytes that direct hepatocyte apoptosis. Manipulation of such apoptosis mechanisms may provide an effective tool to block or suppress liver stage progression and confer disease protection.

The malaria liver stage infection is a current target for identification of protective responses based on the killing activity of parasite-specific CD8 T cells (37) operating independently of bone marrow-derived antigen-presenting cells (APCs) (38). The mechanisms of apoptosis of infected hepatocytes here described have potential impact in building up immunological memory against parasite epitopes presented by MHC class I molecules. On the one hand, phagocytosis of infected hepatocytes apoptotic bodies by professional APCs may represent an additional route to prime CD8 T cells specific for malaria liver stage epitopes (39). On the other hand, responses that increase apoptosis resistance of infected hepatocytes may provide an opportunity for enhancing antigen presentation in the liver, with consequent activation of liver-resident CD8 $\mathrm{T}$ cells that prevent transition to blood stage infection.

\section{AUTHOR CONTRIBUTIONS}

LG and CP-G conceived the study and wrote the manuscript; LG designed, performed, analyzed, and interpreted data from all the experiments; JR, LR-D, and LM participated in the 
design, acquisition, analysis, and interpretation of data relative to FACs and ELISA experiments; CP-G interpreted the data. All the authors critically revised and approved the version to be published.

\section{ACKNOWLEDGMENTS}

The authors are grateful to Maria Mota laboratory for sharing reagents used in this work and for insightful discussions. They also thank Rui Gardner and Telma Lopes from the IGC FACS facility for technical assistance with cell sorting. CP-G is an Affiliated Member of the European Virtual Institute of Malaria Research (EVIMaLaR).

\section{REFERENCES}

1. WHO. WHOMalaria Report 2014. Geneva: WHO (2014). doi:10.1007/s00108013-3390-9

2. Sachs J, Malaney P. The economic and social burden of malaria. Nature (2002) 415:680-5. doi:10.1038/415680a

3. Shin SCJ, Vanderberg JP, Terzakis JA. Direct infection of hepatocytes by sporozoites of Plasmodium berghei. J Protozool (1982) 29:448-54. doi:10.1111/ j.1550-7408.1982.tb05431.x

4. Prudêncio M, Rodriguez A, Mota MM. The silent path to thousands of merozoites: the Plasmodium liver stage. Nat Rev Microbiol (2006) 4:849-56. doi:10.1038/nrmicro1529

5. Miller JL, Sack BK, Baldwin M, Vaughan AM, Kappe SHI. Interferon-mediated innate immune responses against malaria parasite liver stages. Cell Rep (2014) 7:436-47. doi:10.1016/j.celrep.2014.03.018

6. Liehl P, Zuzarte-Luís V, Chan J, Zillinger T, Baptista F, Carapau D, et al. Hostcell sensors for Plasmodium activate innate immunity against liver-stage infection. Nat Med (2014) 20:47-53. doi:10.1038/nm.3424

7. Gonçalves LA, Rodrigues-Duarte L, Rodo J, Vieira de Moraes L, Marques I, Penha-Gonçalves C. TREM2 governs Kupffer cell activation and explains belr1 genetic resistance to malaria liver stage infection. Proc Natl Acad Sci U S A (2013) 110:19531-6. doi:10.1073/pnas.1306873110

8. Heussler V, Rennenberg A, Stanway R. Host cell death induced by the egress of intracellular Plasmodium parasites. Apoptosis (2010) 15:376-85. doi:10.1007/ s10495-009-0435-6

9. Kaushansky A, Ye AS, Austin LS, Mikolajczak SA, Vaughan AM, Camargo N, et al. Suppression of host p53 is critical for Plasmodium liver-stage infection. Cell Rep (2013) 3:630-7. doi:10.1016/j.celrep.2013.02.010

10. Kaushansky A, Metzger PG, Douglass AN, Mikolajczak SA, Lakshmanan V, Kain HS, et al. Malaria parasite liver stages render host hepatocytes susceptible to mitochondria-initiated apoptosis. Cell Death Dis (2013) 4:e762. doi:10.1038/cddis.2013.286

11. Mota MM, Pradel G, Vanderberg JP, Hafalla JC, Frevert U, Nussenzweig RS, et al. Migration of Plasmodium sporozoites through cells before infection. Science (2001) 291:141-4. doi:10.1126/science.291.5501.141

12. Coppi A, Tewari R, Bishop JR, Bennett BL, Lawrence R, Esko JD, et al. Heparan sulfate proteoglycans provide a signal to Plasmodium sporozoites to stop migrating and productively invade host cells. Cell Host Microbe (2007) 2:316-27. doi:10.1016/j.chom.2007.10.002

13. Carrolo M, Giordano S, Cabrita-Santos L, Corso S, Vigário AM, Silva S, et al. Hepatocyte growth factor and its receptor are required for malaria infection. Nat Med (2003) 9:1363-9. doi:10.1038/nm947

14. Leirião P, Albuquerque SS, Corso S, van Gemert G-J, Sauerwein RW, Rodriguez A, et al. HGF/MET signalling protects Plasmodium-infected host cells from apoptosis.CellMicrobiol(2005)7:603-9.doi:10.1111/j.1462-5822.2005.00490.x

15. Tao D, King JG, Tweedell RE, Jost PJ, Boddey JA, Dinglasan RR. The acute transcriptomic and proteomic response of HC-04 hepatoma cells to hepatocyte growth factor and its implications for Plasmodium falciparum sporozoite invasion. Mol Cell Proteomics (2014) 13:1153-64. doi:10.1074/ mcp.M113.035584

\section{FUNDING}

This work was supported by the Fundação para a Ciência e Tecnologia (FCT) through grant POCI/SAU-IMI/61057/2004. LG, JR, and LR-D were supported by FCT fellowships (BD/ 44208/2008, BD/29862/2006, BD/33566/2008, and BPD/44486/ 2008, respectively).

\section{SUPPLEMENTARY MATERIAL}

The Supplementary Material for this article can be found online at http://journal.frontiersin.org/article/10.3389/fimmu. 2017.00090/full\#supplementary-material.

16. Kaushansky A, Kappe SHI. The crucial role of hepatocyte growth factor receptor during liver-stage infection is not conserved among Plasmodium species. Nat Med (2011) 17:1180-1. doi:10.1038/nm.2456

17. Gonçalves LA, Vigário AM, Penha-Gonçalves C. Improved isolation of murine hepatocytes for in vitro malaria liver stage studies. Malar J (2007) 6:169. doi:10.1186/1475-2875-6-169

18. Franke-Fayard B, Trueman H, Ramesar J, Mendoza J, van der Keur $\mathrm{M}$, van der Linden $\mathrm{R}$, et al. A Plasmodium berghei reference line that constitutively expresses GFP at a high level throughout the complete life cycle. Mol Biochem Parasitol (2004) 137:23-33. doi:10.1016/j.molbiopara. 2004.04.007

19. Ishino T, Yano K, Chinzei Y, Yuda M. Cell-passage activity is required for the malarial parasite to cross the liver sinusoidal cell layer. PLoS Biol (2004) 2:E4. doi:10.1371/journal.pbio.0020004

20. Ozaki L, Gwadz R, Godson G. Simple centrifugation method for rapid separation of sporozoites from mosquitoes. J Parasitol (1984) 70:831-3. doi: $10.2307 / 3281779$

21. Goossens PL, Jouin H, Marchal G, Milon G. Isolation and flow cytometric analysis of the free lymphomyeloid cells present in murine liver. J Immunol Methods (1990) 132:137-44. doi:10.1016/0022-1759(90)90407-M

22. Bruña-Romero O, Hafalla JC, González-Aseguinolaza G, Sano G, Tsuji M, Zavala F. Detection of malaria liver-stages in mice infected through the bite of a single Anopheles mosquito using a highly sensitive real-time PCR. Int J Parasitol (2001) 31:1499-502. doi:10.1016/S0020-7519(01)00265-X

23. Kinoshita T, Tashiro K, Nakamura T. Marked increase of HGF mRNA in non-parenchymal liver cells of rats treated with hepatotoxins. Biochem Biophys Res Commun (1989) 165:1229-34. doi:10.1016/0006-291X(89)92733-2

24. Noji S, Tashiro K, Koyama E, Nohno T, Ohyama K, Taniguchi S, et al Expression of hepatocyte growth factor gene in endothelial and Kupffer cells of damaged rat livers, as revealed by in situ hybridization. Biochem Biophys Res Commun (1990) 173:42-7. doi:10.1016/S0006-291X(05)81018-6

25. Maher JJ. Cell-specific expression of hepatocyte growth factor in liver. J Clin Invest (1993) 91:2244-52. doi:10.1172/JCI116451

26. Han J-W, Zhan X-R, Li X-Y, Xia B, Wang Y-Y, Zhang J, et al. Impaired PI3K/ Akt signal pathway and hepatocellular injury in high-fat fed rats. World J Gastroenterol (2010) 16:6111-8. doi:10.3748/wjg.v16.i48.6111

27. Hatano E, Brenner DA. Akt protects mouse hepatocytes from TNF-alpha and Fas-mediated apoptosis through NK- B activation. Am J Physiol Gastrointest Liver Physiol (2001) 281:G1357-68.

28. Matteucci E, Modora S, Simone M, Desiderio MA. Hepatocyte growth factor induces apoptosis through the extrinsic pathway in hepatoma cells: favouring role of hypoxia-inducible factor-1 deficiency. Oncogene (2003) 22:4062-73. doi:10.1038/sj.onc.1206519

29. Ng S, March S, Galstian A, Hanson K, Carvalho T, Mota MM, et al. Hypoxia promotes liver-stage malaria infection in primary human hepatocytes in vitro. Dis Model Mech (2014) 7:215-24. doi:10.1242/dmm.013490

30. Zhao Y, Difrancesca D, Wang XUE, Zarnegar R, Michalopoulos GK, Yin X. Promotion of Fas-mediated apoptosis in type II cells by high doses of hepatocyte growth factor bypasses the mitochondrial requirement. J Cell Physiol (2007) 213:556-63. doi:10.1002/JCP 
31. Yin XM, Wang K, Gross A, Zhao Y, Zinkel S, Klocke B, et al. Bid-deficient mice are resistant to Fas-induced hepatocellular apoptosis. Nature (1999) 400:886-91. doi:10.1038/23730

32. Hu S, Vincenz C, Ni J, Gentz R, Dixit VM. I-FLICE, a novel inhibitor of tumor necrosis factor receptor-1- and CD-95-induced apoptosis. J Biol Chem (1997) 272:17255-7. doi:10.1074/jbc.272.28.17255

33. Lindroos PM, Zarnegar R, Michalopoulos GK. Hepatocyte growth factor (hepatopoietin A) rapidly increases in plasma before DNA synthesis and liver regeneration stimulated by partial hepatectomy and carbon tetrachloride administration. Hepatology (1991) 13:743-50. doi:10.1002/hep.1840130422

34. Huh C-G, Factor VM, Sánchez A, Uchida K, Conner EA, Thorgeirsson SS. Hepatocyte growth factor/c-met signaling pathway is required for efficient liver regeneration and repair. Proc Natl Acad Sci U S A (2004) 101:4477-82. doi:10.1073/pnas.0306068101

35. Grzelakowska-Sztabert B, Dudkowska M. Paradoxical action of growth factors: antiproliferative and proapoptotic signaling by HGF/c-MET. Growth Factors (2011) 29:105-18. doi:10.3109/08977194.2011.585609

36. Douglass AN, Kain HS, Abdullahi M, Arang N, Austin LS, Mikolajczak SA, et al. Host-based prophylaxis successfully targets liver stage malaria parasites. Mol Ther (2015) 23:857-65. doi:10.1038/mt.2015.18
37. Holz LE, Fernandez-Ruiz D, Heath WR. Protective immunity to liver-stage malaria. Clin Transl Immunol (2016) 5:e105. doi:10.1038/cti.2016.60

38. Chakravarty S, Cockburn IA, Kuk S, Overstreet MG, Sacci JB, Zavala F. CD8+ T lymphocytes protective against malaria liver stages are primed in skin-draining lymph nodes. Nat Med (2007) 13:1035-41. doi:10.1038/nm1628

39. Leiriao P, Mota MM, Rodriguez A. Apoptotic Plasmodium-infected hepatocytes provide antigens to liver dendritic cells. J Infect Dis (2005) 191:1576-81. doi:10.1086/429635

Conflict of Interest Statement: The authors declare that the research was conducted in the absence of any commercial or financial relationships that could be construed as a potential conflict of interest.

Copyright (C) 2017 Gonçalves, Rodo, Rodrigues-Duarte, de Moraes and PenhaGonçalves. This is an open-access article distributed under the terms of the Creative Commons Attribution License (CC BY). The use, distribution or reproduction in other forums is permitted, provided the original author(s) or licensor are credited and that the original publication in this journal is cited, in accordance with accepted academic practice. No use, distribution or reproduction is permitted which does not comply with these terms. 